\title{
DE FÉTICHE À MARQUEUR D'UNE CÉLÉBRITÉ PASSÉE : LA CARTE DE HOCKEY COMME OBJET AU CEUR D'UNE CULTURE MATÉRIELLE NOSTALGIQUE
}

\author{
Fannie Valois-Nadeau ${ }^{1}$
}

\begin{abstract}
Cet article s'intéresse à la "vieille » carte de hockey de Léo Gravelle, un ancien joueur du club de hockey Canadien de Montréal, de même qu'aux pratiques culturelles réalisées par son entremise. Par le biais d'une approche inspirée des cultural memory studies - et particulièrement celles qui ont développé des réflexions sur la matérialité -, il s'agit d'identifier les différentes trajectoires de la carte et d'interroger comment le passé de cette ancienne célébrité y est réactualisé. En étant la fois un objet de mémoire, un mémorial vivant et un marqueur d'une célébrité passée, la carte de hockey de Léo Gravelle contribue à chaque fois qu'elle est mobilisée, à le rendre encore là et surtout encore signifiant en tant qu'ancien joueur de hockey vieillissant. À partir d'une analyse des événements publics où cette carte de hockey a refait surface, cet article met en évidence la façon dont cet objet incarne, à l'échelle d'un individu, une culture sportive nostalgique déjà bien établie.
\end{abstract}

Le milieu du hockey professionnel nord-américain, et plus particulièrement celui de l'équipe du Canadien de Montréal, est marqué par une culture nostalgique, qui prend forme à travers une série de commémorations, rétrospectives et intronisations (Valois-

1 Fannie Valois-Nadeau est $\mathrm{PhD}$ en communication, chercheure en études culturelles et chargée de cours à l'Université du Québec en Outaouais.

Recherches en communication, $n^{\circ} 46$ - Article publié le 26/06/2018 
Nadeau, 2014). Ces événements, où les prouesses passées de l'équipe sont à l'honneur, ponctuent régulièrement le calendrier sportif et constituent comme le suggèrent Stephen P. Andon et Davis W. Houck, des « elements of political economy and commodification of nostalgia worked together to create a sporting spectacle » (2011, p. 3). Ces célébrations, chères au spectacle sportif contemporain, prennent forme à partir d'une culture matérielle dont l'esthétique et le contenu font office de rappel des débuts du spectacle sportif. Qu'ils soient archives, mémorial ou souvenirs spécialement mis en vente pour l'occasion, ces objets matérialisent et alimentent à leur manière cette tendance à la nostalgie de même qu'à la valorisation des formes culturelles du passé.

Parmi tous les objets constitutifs de la culture matérielle nostalgique du hockey, les cartes de sport occupent une position singulière. En effet, en plus de leurs valeurs monétaires et affectives, ces cartes font partie du sport-spectacle et de la culture nordaméricaine depuis des décennies. À la manière des autres cartes issues du sport professionnel, les cartes de hockey ont traversé les années et les modes pour meubler le quotidien de nombreux enfants (surtout chez les garçons, mais pas exclusivement), mais aussi celui des collectionneurs et des fans les plus aguerris. À la fois banales et précieuses, faisant partie des jeux et des loisirs de générations d'enfants, mais également des lucratifs encans destinés aux collectionneurs professionnels, les cartes de hockey sont marquées des logiques du milieu du divertissement duquel elles sont nées. Toutes n'ont pas la même valeur, que ce soit au plan symbolique ou au moment de l'échange, mais toutes s'inscrivent néanmoins dans une culture matérielle dont l'ancrage historique leur confère une valeur spécifique.

Cet article s'intéresse plus particulièrement aux différents statuts et pratiques culturelles qui adviennent par le biais d'une carte en particulier, soit celle de Léo Gravelle ${ }^{2}$, ancien joueur du Canadien de Montréal de 1946 à 1951 (voir Figure 1). Cette carte

2 Léo Gravelle s'est malheureusement éteint six mois après notre entrevue, soit en octobre 2013. Je profite de l'occasion pour remercier Léo et son fils Denys de m'avoir accordé cette entrevue. Il était âgé de 87 ans au moment de notre rencontre. 
fut bien plus qu'un objet souvenir permettant la représentation et la reconnaissance d'une époque révolue ; par son usage et sa circulation récente, elle a mis en évidence d'autres pratiques culturelles que celles généralement attendues à travers le hobby de collection ou l'expression d'un fandom. Inspiré des cultural memory studies et des études culturelles sur le hockey, cet article interroge les relations au passé (parfois nostalgiques) qui se forment en regard de cet objet. Bien qu'elle partage le caractère sériel et l'inscription au sein de visées lucratives qui caractérisent de nombreux objets rétros (Hallegate, 2013), la carte de hockey de Léo Gravelle et les pratiques qui adviennent par sa circulation ne se limitent pas à la répétition figée d'un passé, comme semble parfois le sous-entendre l'expression « rétro » telle qu'utilisée par Simon Reynolds (2011). La présence relativement récente de cette « vieille » carte de hockey a plutôt permis d'envisager la création de nouvelles relations et rapports imprévus à l'objet ancien. En s'apparentant davantage à ce que Svetlana Boym (2001) qualifie de « nostalgie réflexive », pour évoquer " a new flexibility, not the re-establishment of stasis » (2001, p. 49), les pratiques qui ont fait circuler la carte de Léo Gravelle ont plutôt contribué à rendre cet ancien joueur vieillissant encore présent et surtout encore signifiant.

L'analyse qui suit se base sur une entrevue réalisée avec Léo Gravelle et son fils Denys (2014) $)^{3}$ et une recension médiatique des événements où cette carte a fait l'objet d'une attention publique. Une fête donnée en son honneur au sein de sa résidence pour personnes âgées, le recours à sa vieille carte par une jeune équipe de hockey, de même que l'annonce de son décès ont été autant d'événements (ayant eu lieu de 2008 à 2013) au sein desquels il fut fait mention de cette carte. Les productions médiatiques sélectionnées incluent principalement des articles de presse (principalement issus de journaux régionaux) et deux enregistrements vidéos de sa participation à des émissions télévisées. Ces documents ont ainsi servi de base pour mener une

3 Cette entrevue a eu lieu dans le cadre d'une thèse doctorat (Valois-Nadeau, 2014). 
analyse de discours visant à retracer la circulation récente de sa carte et les diverses façons dont elle est (ré)appropriée.

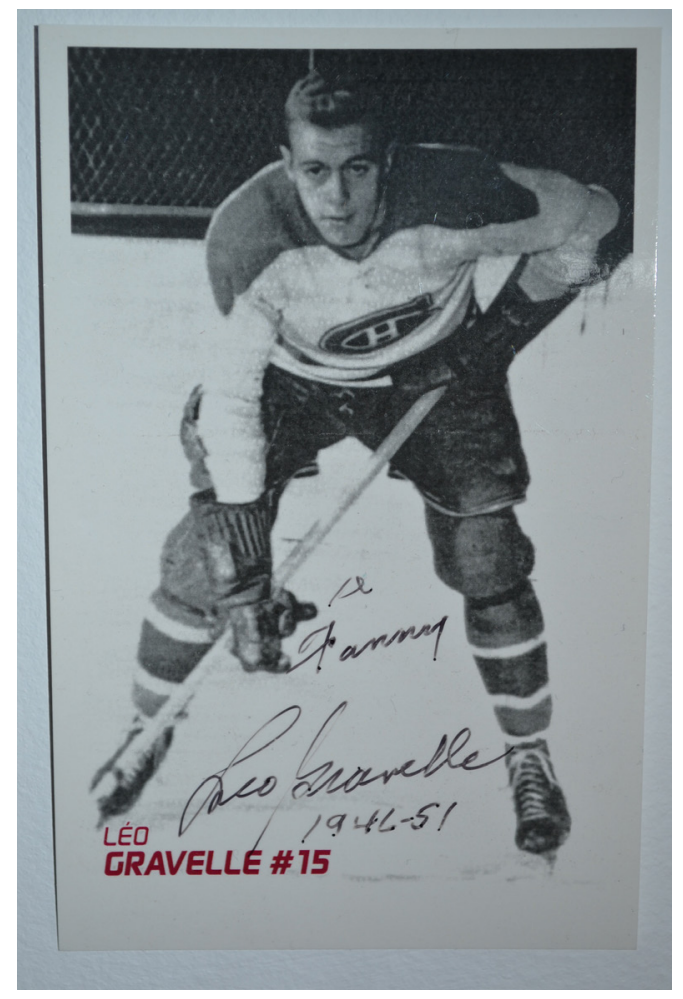

Figure 1. Photographie de la carte de Léo Gravelle, 2012 Source : archives personnelles (Valois-Nadeau, 2014)

La pluralité des lieux où la carte est apparue et des sources mobilisées permet ainsi de rompre avec une conception « immobile » de la nostalgie et ouvre l'analyse de relations imprévues au passé. Cette carte de hockey devient alors une porte d'entrée privilégiée pour interroger comment s'incarne et se réarticule, à l'échelle d'un individu, la culture nostalgique qui caractérise l'univers du Canadien de Montréal (Bélanger, 2000), de même que les nouvelles pratiques qui en émanent. Les retracer met ainsi en évidence les différents statuts joués par cette même carte, qui est tour à tour devenue technologie de mémoire (Clermont, 2009), mémorial vivant (Allen \& Brown, 2011), 
fétiche (Fernandez et Lastovicka, 2011) de même qu'un marqueur de célébrité quelque peu oubliée.

\section{La carte de hockey comme technologie de mémoire commercialisée}

Plus qu'un objet de loisir et qu'un divertissement destiné aux enfants, les cartes de sport constituent l'un des premiers lieux où furent conservées les statistiques des joueurs, sans toutefois avoir été créées à cette fin. L'apparition des premières cartes de sport à la fin du $19^{\mathrm{e}}$ siècle a en effet coïncidé avec l'émergence des marchés de consommation de masse, et a depuis toujours été liée à des fonctions de marketing. Ainsi, les cartes de sport ont d'abord été créées pour être glissées à l'intérieur de paquets de cigarettes, d'emballages de friandises et de produits d'alimentation pour stimuler les ventes et attirer de nouveaux consommateurs (Hewitt, 2005). D'emblée amalgamées à des aspirations commerciales, les cartes de hockey n'ont cessé d'être approchées comme un objet promotionnel intégré à une logique marchande.

En dépit de son caractère intrinsèquement commercial, la carte de sport - par les informations qu'elle contient - a joué un rôle déterminant dans le développement d'une culture sportive centrée sur la connaissance et l'expertise. À la manière d'une technologie de mémoire, qui, comme le suggère Patricia Clermont (2010) fait être et donne corps aux représentations du passé, la carte a rendu possible l'archivage de statistiques et du même coup, la production de vedettes sportives. En permettant de se remémorer les performances des joueurs tout en les comparant et les classant, la carte de sport a ainsi contribué à l'essor d'une culture sportive centrée sur la mesure des prouesses et la connaissance statistique - et ce, avant même le développement d'une presse sportive qui en redéfinira les contours et les moyens de visibilité.

Malgré le fait qu'elles soient produites en série et de qualité parfois discutable, la valeur accordée aux cartes de sport - qu'elle soit monétaire ou symbolique - en a fait des objets qui comptent, pour reprendre les propos de Philippe Le Guern (2009), par lesquels se sont développées au fil du temps des relations signifiantes et 
affectives. Comme l'ont documenté Richard Gruneau et David Whitson (1993), l'engouement pour le hockey au Canada s'est justement forgé au croisement de la consommation de ces produits et d'une affiliation commanditaire commerciale de premier plan. C'est également dans ce contexte qu'une économie des cartes de hockey s'est développée, où l'échange et la vente se sont organisés autour de balises qui attribuent généralement une grande valeur aux spécimens rares et « vieux ». Sans se départir de leur caractère marchand, les cartes de hockey ont plutôt été intégrées avec les années dans un réseau économique plus ou moins formalisé, basé sur la valorisation d'éléments passés. Comme en témoigne l'ancien joueur de hockey Jean Béliveau au sein de son autobiographie :

Les expositions de cartes et séances de signature attirent des milliers de passionnés et de collectionneurs qui sont prêts à payer pour rencontrer certains joueurs. Les organisateurs de ces expositions versent même un cachet aux athlètes qui acceptent avec plaisir de venir signer cartes, rondelles, bâtons chandails et les nombreux autres articles que leur apportent les amateurs ou qui sont vendus sur place. (Béliveau, Goyens, \& Turowetz, 2005, p. 339)

En dépit du fait qu'elle s'apparente à un vieil objet de par sa photo en noir et blanc, la carte de Léo Gravelle ne représente toutefois pas le type de produits recherchés au sein de ces salons. En plus de la renommée plutôt locale de l'ancien joueur de hockey (qui pèse dans la valeur qui sera attribuée à la carte), son format n'est pas conforme aux standards habituels ${ }^{4}$. Malgré son apparence « ancienne », la carte de Léo Gravelle est tirée d'une série récemment produite par le Club de hockey du Canadien spécialement consacrée (et destinée) à ses anciens joueurs. Les pixels bien visibles de la photographie (signes d'une moins bonne qualité de la carte) indiquent également qu'elle a été créée pour

4 La taille de la carte s'apparente davantage à celle d'une carte postale que des cartes de sport. 
honorer et faire plaisir aux anciens joueurs de l'équipe, plutôt que pour être échangée.

En dépit de leur différence de qualité, les cartes « officielles » et celles consacrées à ces anciens joueurs reprennent les mêmes éléments de la culture matérielle du hockey en portant notamment la trace de ses articulations au milieu de consommation. Par exemple, le logo de Molson Export Ale, un des commanditaires de l'équipe, qui est inséré au verso de la carte rend visibles les liens étroits (voir synergiques, comme le souligne Anouk Bélanger, 2000) qui unissent depuis des décennies le hockey et les grands producteurs de bière. En produisant de telles cartes, l'organisation du Canadien de Montréal a ainsi donné à ses anciens joueurs - même à ceux qui sont inactifs depuis des décennies les moyens d'être encore visibles à travers des objets propres au milieu du sport professionnel. Bien que la carte de Léo Gravelle reprenne à sa manière les normes dominantes de cet univers, cette technologie de mémoire, qui repose spécifiquement sur le maintien de cette culture nostalgique, rend néanmoins présentes son affiliation à l'équipe du Canadien de Montréal de même que son inscription dans le milieu du sport. La carte, dont le visuel est associé aux anciens modèles, de même que l'âge avancé de Léo Gravelle, a créé les conditions propices à une nouvelle forme de circulation de l'objet. En effet, après des années passées plutôt dans l'ombre des grandes vedettes, Léo Gravelle est devenu une sorte de mémorial vivant, et sa carte de hockey, l'objet fétiche de jeunes sportifs.

\section{D'objet fétiche à mémorial vivant}

Loin d'être uniquement définie par l'univers de consommation au sein duquel elle fut produite, la carte de hockey - et particulièrement celle de Léo Gravelle - a acquis différentes valeurs au gré de sa circulation. À la manière d'Arjun Appadurai (1986) qui a questionné la «vie sociale des objets » à travers leur circulation et leurs interactions humaines, il s'agit ici d'identifier en quoi la carte de Léo Gravelle s'est transformée tout en restant balisée et configurée par diverses pratiques culturelles, jamais 
bien loin des codes du hockey. En devenant un objet fétiche, la carte de hockey permet d'interroger les valeurs et les statuts qu'elle acquiert en dehors des relations commerciales. Cette carte, qui a matérialisé du même coup la valeur sociale accordée à cette formation sportive, a également contribué à faire de l'ancien joueur un «mémorial vivant » qui incarne le passé glorieux de l'équipe du Canadien de Montréal. Approché par Matthew Allen et Stephen D. Brown (2011) comme un assemblage de gens, de corps, d'objets et de récits, le mémorial vivant (living memorial) représente des réseaux complexes à travers lesquels les liens au passé se déclinent et se réactualisent ensemble. C'est ainsi que le corps de Léo Gravelle, sa carte et les récits entourant la victoire d'une jeune équipe de hockey ont ainsi mis en évidence la prégnance (et lourdeur) d'une culture du hockey fondée sur le maintien de ses traditions.

Lors d'un tournoi de hockey en $2009^{5}$, une jeune équipe de la ville d'Amos ${ }^{6}$ avait pris l'habitude d'afficher derrière le banc des joueurs la « vieille » carte de Léo. À la manière des représentations graphiques religieuses affichées pour assurer protection et sécurité, la carte de Léo Gravelle a été collée et recollée lors de chaque match disputé par ces joueurs âgés de 13 et 14 ans. Bien que peu connu et médiatisé jusqu'alors, le nom de Léo Gravelle est devenu, au cours de ce tournoi, leur cri de ralliement, chanté tout juste avant le début des parties dans le but de fédérer et stimuler le groupe. " Comme dans un conte de fées », tel que le suggérait un animateur de télévision qui a couvert l'événement (Paradis, 2009), les défaites qu'accumulait jusqu'alors l'équipe ont été remplacées par des victoires, permettant du même coup à l'équipe d'accéder au grand tournoi de fin de saison. Afin de poursuivre cette séquence victorieuse et de maximiser leurs chances de succès, les jeunes joueurs ont invité Léo Gravelle à assister à la grande finale - ce qu'il fit - malgré la distance

5 Cette année correspond au centenaire de l'équipe de hockey du Canadien de Montréal et fut particulièrement marquée par de nombreuses commémorations et célébrations du passé. Il n'est donc pas étonnant que la « vieille » carte de Léo ait refait surface au cours de cette période.

6 La ville d'Amos est située à environ 5 heures 30 de voiture de la résidence de Léo Gravelle. 
géographique qui les séparait et sa fatigue physique. Comme l'a raconté $\mathrm{M}$. Turcotte, un des parents responsables de l'équipe, à un journaliste local :

Il [Léo] est venu voir les joueurs dans la chambre, il leur a donné des cartes de hockey autographiées et des pièces de $1 \$$ du Centenaire du Canadien. Il leur a fait un petit speech. Il était vraiment ému, il y avait longtemps qu'il n'avait pas eu ce genre d'attention, surtout de la part de jeunes joueurs (Guindon, 2009).

À travers ce tournoi, Léo Gravelle et sa carte de hockey sont ainsi devenus l'emblème et l'objet porte-bonheur d'une équipe, les éloignant tous deux de leurs usages et rôles habituels. Empruntant les codes du fétiche qui, dans la tradition anthropologique, réfère aux « objects that are taken out of ordinary use, enshrined in public or private collections, and displayed for their exhibition value " (Fernandez \& Lastovicka, 2011, p. 278), la carte de hockey devint l'objet par lequel se développa une relation « mystique ». À travers ces pratiques maintes fois réitérées, la carte de hockey et la présence de Léo Gravelle sont devenues des moyens de garantir le succès de cette jeune équipe. Dans une culture très superstitieuse et ritualisée comme celle du hockey sur glace (Robidoux, 2001), leur présence allait tout à fait de soi. En effet, dans un milieu où les mêmes gestes peuvent être répétés mécaniquement sur une base régulière dans le but de préserver l'unité de l'équipe et favoriser la victoire, la fétichisation de la carte de hockey a mis en lumière l'importance des rituels et du recours aux traditions passées sur lesquelles s'est bâtie la culture du hockey. Ces pratiques sont présentes même chez les jeunes joueurs encore bien loin de mener une carrière professionnelle. Entretenue par les médias qui couvrent et diffusent ces événements, la culture nostalgique du hockey se maintient par l'intégration régulière et normalisée d'éléments référant à divers passés. En s'éloignant d'une conception marxisante du terme (qui prétendrait à un attachement faussé à l'objet), la fétichisation de la 
carte de hockey rend plutôt saillante la réappropriation de ce vieil objet populaire qui semble ainsi être encore grandement valorisé.

Dans le contexte où elles ont été mobilisées, la "vieille » carte de Léo Gravelle de même que sa propre présence physique ont été sollicitées pour leur apport à une future victoire plutôt que pour leur potentiel de réminiscence ou de représentation du passé. À l'importance qu'occupe le rituel au sein de cette culture nostalgique s'ajoute alors celle de la présence d'anciens joueurs ayant partagé la passion d'un jeu qui n'a cessé d'évoluer ainsi que l'intergénérationalité supposée exister entre ces générations de joueurs pourtant très différentes. C'est donc pour reprendre ces manières de faire communes au milieu que Léo Gravelle a spontanément prodigué quelques conseils aux joueurs avant le match, et ce, bien qu'il soit sceptique des effets de ses propres commentaires sur le sort de la partie et le développement des aptitudes des jeunes joueurs. Lors de notre entretien, Léo Gravelle affirmait que "quand ben même tu leur dis quelque chose, ils oublient tout avant de jouer ... [mais] y'ont été motivés de m'voir là ».

La carte et le corps vieillissant de Léo semblent alors plutôt avoir été les traces matérielles nécessaires à la performance du rituel et de la tradition tant valorisés au sein de cette culture nostalgique, plutôt que des outils pour améliorer leur compréhension du jeu ou accroître leur connaissance du milieu du hockey des années 1940 et 1950. À l'image d'un mémorial vivant (Allen et Brown, 2011), Léo Gravelle et sa carte ont semblé incarner un passé particulier, perceptible à travers les formes matérielles de son corps et du papier, et au sein des gestes et habitudes répétés. Bien qu'il ait été peu écouté, le discours d'avant-match de l'ancien joueur a importé, ne serait-ce que pour l'enthousiasme et la motivation générés, comme le suggérait $M$. Gravelle. Même si le récit du passé fut au final peu entendu, voire même fétichisé, le retour « public » du corps âgé de cet ancien joueur de hockey et de cette " vieille » carte leur a permis de continuer d'acquérir une valeur. À l'instar d'Arjun Appadurai (1986) qui voyait dans l'échange et la circulation le moment où la valeur des objets se définit, cette carte de hockey - et ce malgré son caractère hors norme - et a vu 
sa valeur être redéfinie grâce à sa circulation et son appropriation. Cette valeur a continué de se transformer, notamment lorsque la carte contribue à le maintenir célèbre.

\section{La carte de hockey comme marqueur de célébrité quotidien}

Denys [fils de Léo Gravelle] : Léo, ses cartes de hockey, il les donne.

Léo : J'en donne.

Denys : Il en a à profusion, donc il les donne. Le Canadien les fournit... et puis, les gens qui sont alentour de lui, au début, sont ben contents d'avoir ça...

M. Gravelle m'a reçue en 2012 dans son appartement accompagné de son fils Denys pour me raconter les souvenirs de son passage avec le club de hockey du Canadien de Montréal. À la fin de notre entretien (et ce, sans que j'en fasse la demande), Léo Gravelle est allé chercher une de ses cartes de hockey et l'a signée et dédicacée à mon nom. Ce geste s'est fait comme un automatisme, comme une vieille habitude répétée maintes fois au fil du temps. Ainsi, même si sa dernière présence sur la glace avec ce club de hockey remonte à plus de 60 ans, Léo Gravelle conserve à portée de mains - et précieusement - ses nombreuses cartes qui lui permettent du même coup de réaliser ce geste généralement réservé aux célébrités. Plus qu'un objet qui atteste ses anciennes performances sportives, le paquet de cartes spécialement créé à son effigie lui a permis de s'inscrire dans un milieu hors du commun et de réaffirmer son parcours privilégié, et ce, même s'il prit fin il y a plus d'un demi-siècle et qu'il demeure en marge des grandes commémorations de l'équipe de hockey (Valois-Nadeau, 2014). Agissant tel un marqueur de célébrité, la carte lui a attribué une notoriété quelque peu oubliée, car bien qu'il ait été membre de l'équipe sportive la plus titrée et adulée au Québec et qu'il joua aux côtés de Maurice Richard - considéré comme l' '« idole d'un peuple » (Melançon, 2016) - sa carrière a peu fait les manchettes et demeure peu intégrée aux grands récits entourant l'équipe. Le passé singulier de Léo Gravelle rayonnait davantage auprès de 
ses voisins, amis et famille, faisant de lui une célébrité à l'échelle locale.

Toutefois, avec la production récente de ces « vieilles » cartes de hockey, l'organisation du Canadien de Montréal a fourni à Léo Gravelle les moyens de rendre public une forme de notoriété en rendant surtout visible son affiliation au club. Devenu en position de faire don, Léo Gravelle a pu jusqu'aux derniers moments de sa vie se construire un statut et capital social particulier, comme l'a suggéré Andrew Baker (2012) au cours de ses travaux sur les échanges d'objets dans les communautés de fans de rock. La distribution de cartes, dont le rôle est ici à mi-chemin entre la carte d'affaires et la signature d'autographes, a en effet permis à Léo Gravelle de faire reconnaître son allégeance au groupe d'anciens joueurs du club, et ce, même si la majorité de ses coéquipiers étaient alors décédés et qu'il entretenait peu de liens avec les nouvelles générations de retraités. Jusqu'aux derniers jours de sa vie, Léo Gravelle distribuait encore sur une base régulière sa carte de joueur de hockey. Dans l'article de journal qui a annoncé son décès au public local, le journaliste Martin Comptois a justement fait état de cette pratique de distribution de cartes :

L'octogénaire avait participé au dévoilement de la statue de Guy Lafleur à Thurso, il y a un mois et demi. Sa présence avait été soulignée parmi les anciens joueurs du Canadien sur place. « Il avait remis plusieurs de ses cartes, a relaté Pierre Gravelle. Sa dernière carte, il l'a justement donnée hier (mardi) soir à $18 \mathrm{~h}$. Il avait encore un effet quand il rentrait dans une place. Il a toujours été un fier ambassadeur du Canadien ». (Comptois, 2013)

La circulation continue de cette carte de hockey a donc contribué à inscrire Léo Gravelle au sein de cette culture particulière, réservée aux athlètes professionnels médiatisés et vedettes populaires. À travers le don et la signature de cartes, maintes fois répétés, l'attachement à ce passé s'est cristallisé au sein même de cet objet produit en série.

$\mathrm{Au}$ même titre que ses trophées, ses souvenirs et ses photos affichés en plein centre de son appartement, les cartes de hockey 
à son effigie ont contribué à faire vivre ce passé et à le maintenir présent de manière quotidienne. Cette façon de vivre près de ses objets souvenirs, avec eux, et le soin qu'il leur attribuait rendent compte d'une forme d'assemblage qui s'est créé à travers le temps entre Léo Gravelle et ses objets. Comme le souligne Martjee Hoogsteyns (2013) à travers l'exemple des ballerines qui deviennent l'extension du corps des danseuses, les relations intimes qui se développent entre les sujets et certains objets signifiants au fil des années participent à (trans)former les uns comme les autres. Ces objets, dont semble faire partie la carte de hockey, ne font donc pas que meubler l'appartement ou contenir des souvenirs; ils participent par le biais des gestes et de leurs mouvements à la constitution d'une nouvelle entité, qui dépasse les simples caractéristiques de la personne ou la chose prise séparément. (Re)devenu célébrité par leur entremise, le parcours de Léo Gravelle s'est alors constitué grâce à cette écologie matérielle singulière et à sa mobilisation constante. L'assemblage non-dualiste des personnes et des choses que décrit Hoogsteyns met alors en évidence l'importance cruciale de ces objets, la longévité de leurs relations, de même que leur signification, qui a tenu jusqu'au dernier moment. Ainsi, sans pouvoir réduire l'un à l'autre, Léo Gravelle et ses objets souvenirs, qui sont centraux et constitutifs de la culture du hockey, sont apparus au fil du temps comme un assemblage « personne-chose » ayant vieilli ensemble.

\section{Conclusion}

Au cours de leur siècle d'existence, les cartes de hockey ont circulé au sein de divers milieux et incarné différents rapports au temps. Mises en forme par les logiques d'un marché de consommation en plein essor et popularisées par une nostalgie entretenue par les organisations sportives, les cartes de hockey sont également issues d'une culture de masse qui a fait naître de nombreux loisirs. Objet de collection, source de renseignements précieux à une époque où le sport était peu médiatisé et forme culturelle par laquelle les fans concrétisent leur attachement, la carte de hockey matérialise différentes relations et pratiques 
culturelles en regard desquelles le milieu du sport professionnel s'est constitué. L'incursion au sein du parcours de celle Léo Gravelle, ne serait-ce qu'en raison de son ancrage historique et ses références singulières au passé, a élargi le spectre des pratiques auxquelles réfèrent généralement cet objet. En proposant une incursion au sein de ces divers statuts de ce « vieil objet ", tel que le fétiche, le mémorial vivant et le marqueur de célébrité, cet article a souhaité explorer l'aspect créatif de cette culture nostalgique tout en soulevant le poids de la culture du hockey dans les manières d'encadrer et de rendre présent ce passé. En effet, bien qu'elles aient été réarticulées à son histoire personnelle, ces pratiques inspirées de celles déjà en vogue dans le milieu ont contribué à réitérer la valeur du Canadien et à poursuivre sa glorification.

Depuis la création du Canadien de Montréal en 1909, de nombreux joueurs (voire la majorité) sont tombés dans l'oubli et n'ont pas pu jouir d'une reconnaissance publique une fois leur carrière terminée. La carte de hockey fabriquée par le Canadien de Montréal, de même que la distribution qu'il en faisait, aura néanmoins permis à Léo Gravelle d'être encore présent au sein de ce milieu qu'il chérissait. En plus de contrer l'oubli de son passage au sein de l'équipe du Canadien de Montréal, les pratiques culturelles qui se sont réalisées par l'entremise de cette carte ont permis la reconnaissance de sa carrière et de son inscription dans un univers sélect. Dans la mesure où, comme le suggèrent Plate et Smelik, « the materiality of memory has a spatial dimension : it literally takes place » (2009, p. 9), Léo Gravelle a ainsi pu « prendre place » au sein de ce milieu grâce à ce « vieil » objet, qui fait sens et importe encore à ce jour. Car si Léo Gravelle n'est plus, sa carte reste présente et continue, elle, à circuler, comme un objet toujours vivant.

En interrogeant la carte de hockey à travers les différentes pratiques et relations à l'objet, cet article a souhaité ouvrir les réflexions sur les cultures matérielles nostalgiques à travers leurs appropriations au présent, bien qu'elles soient encadrées et balisées par des manières de faire popularisées. Cet article invite alors à penser les relations aux objets du passé dans la pratique 
et le geste, dans le « en train de se faire », pour questionner « a materiality that materializes » (Tiainen, Kontturi, \& Hongisto, 2015, p. 5), de même que les cultures de la célébrité, du sport professionnel, de la nostalgie comme celles du vieillissement. Si de récentes approches en études culturelles s'ouvrent aux nouveaux/néo matérialismes pour penser aux matérialités et micro-politiques du quotidien (Tiainen et al., 2015), il serait intéressant que ces questionnements soient davantage introduits au sein des études sur la mémoire et la nostalgie, qui approchent souvent les objets populaires par leur contenu et représentation. Cet intérêt pour les relations à l'objet du passé et cette façon de les interroger à travers les pratiques initiées par Léo Gravelle comme par de jeunes hockeyeurs auront également permis de mettre en évidence le caractère relationnel du vieillissement (Grenier, 2012), où Léo et sa carte ont vécu et vieilli ensemble, à travers sa propre appropriation comme celle des autres.

\section{Références}

Allen, M. J., \& Brown, S. D. (2011). Embodiment and living memorials: The affective labour of remembering the 2005 London bombings. Memory Studies, 4(3), 312-327.

Andon, S. P., \& Houck, D. W. (2011). Spectacularized Sport: Understanding the Invention of a Nostalgic, Commodified Sporting Event. International Journal of Sport Communication, 4 (pp. 1-19).

Appadurai, A. (1986). The Social Life of Things. Commodities in Cultural Perspective. Cambridge : Cambridge University Press.

Baker, A. (2012). The Exchange of Material Culture Among Rock Fans in Online Communities. Information, Communication \& Society, 15(4), pp. 519-536.

Bélanger, A. (2000). Sport Venues and the Spectacularization of Urban Spaces In North America: The Case of the Molson Centre in Montreal. International Review for the Sociology of Sport, 35(3), pp. 378-397.

Béliveau, J., Goyens, C., \& Turowetz, A. (2005). Jean Béliveau. Ma vie Bleu-BlancRouge. Montréal : Éditions Hurtubise HMH.

Clermont, P. (2010). De la mémoire au mémoriel. Analyse de la constitution et de l'effectivité de deux personnalités publiques au Québec. Éléments d'une problématique du mémoriel. Université de Montréal, Montréal.

Comptois, M. (2009, 19 décembre). Oublié par le Canadien lors des célébrations du 100 anniversaire. Le chandail de Gravelle retiré... par ses amis. Le Droit. Disponible à http://www.lapresse.ca/le-droit/sports/lnh/200912/18/01-932497-le-chandail-degravelle-retire-parses-amis.php 
Comptois, M. (2013, 31 octobre). La Gazelle n'est plus. Le Droit. Disponible à http:// www.lapresse.ca/ledroit/sports/canadien/201310/30/01-4705557-leo-gravelle-ditla-gazelle-nest-plus.php

Fernandez, K. V., \& Lastovicka, J. L. (2011). Making Magic: Fetishes in Contemporary Consumption. Journal of Consumer Research, 38(2), pp. 278-299.

Grenier, L. (2012). Ageing and/as enduring: Discussing with « Turtles [that] don't die of old age ». Dans G. Latzko-Toth \& F. Millerand (Éd.), TEM 2012: First conference proceedings of the CCA technology and emerging media track. Disponible à http://www.tem.fl.ulaval.ca/www/wp-content/PDF/Waterloo_2012/GRENIERTEM2012.pdf

Gruneau, R., \& Whitson, D. (1993). Hockey night in Canada. Toronto : Grammond Press.

Guindon, M. (2009, 10 avril). Un ancien Canadien inspire le Carella d'Amos. L'Écho abitibien. Disponible à http://www.lechoabitibien.ca/2009/04/10/un-anciencanadien-inspire-le-carella-damos

Hewitt, J. D. (2005). Race, Performance, and Baseball Card Values. Journal of Sport and Social Issues, 29(4), pp. 411-425.

Hoogsteyns, M. (2013). Giving more weight to the ballerina: Material agency in the world of pointe shoes. International Journal of Cultural Studies, 16(2), pp. 119-133.

Le Guern, P. (2009). « No matter what they do, they can never let you down... » : Entre esthétique et politique : sociologie des fans, un bilan critique. Réseaux, 153(1), p. 19.

Melançon, B. (2006). Les yeux de Maurice Richard. Montréal : Fides.

Paradis, F. (2009, 9 avril). TVAdirect.com, Montréal : TVA. Disponible à https:// myspace.com/365687638/video/entrevue-tva-l-o-gravelle-9-avril-2009/55552314)

Plate, L., \& Smelik, A. (2009). Technologies of memory in the arts. Basingstoke : Palgrave Macmillan.

Reynolds, S. (2011). Retromania. Pop Culture's Addiction to its Own Past. New York : Faber and Faber.

Robidoux, M. (2001). Men at play. A working understanding of professional hockey (McGill-Queen's University Press). Montréal : Kingston.

Tiainen, M., Kontturi, K.-K., \& Hongisto, I. (2015). Preface. Cultural Studies Review, 21(2), p. 4.

Valois-Nadeau, F. (2014). Un centenaire, des faire mémoire. Analyse des pratiques de mémoire autour du Canadien de Montréal. Université de Montréal, Montréal.

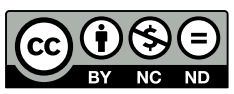

Publié sous la licence Creative Commons

«Attribution - Pas d'Utilisation Commerciale - Pas de Modification 4.0 International» (CC BY-NC-ND) 